
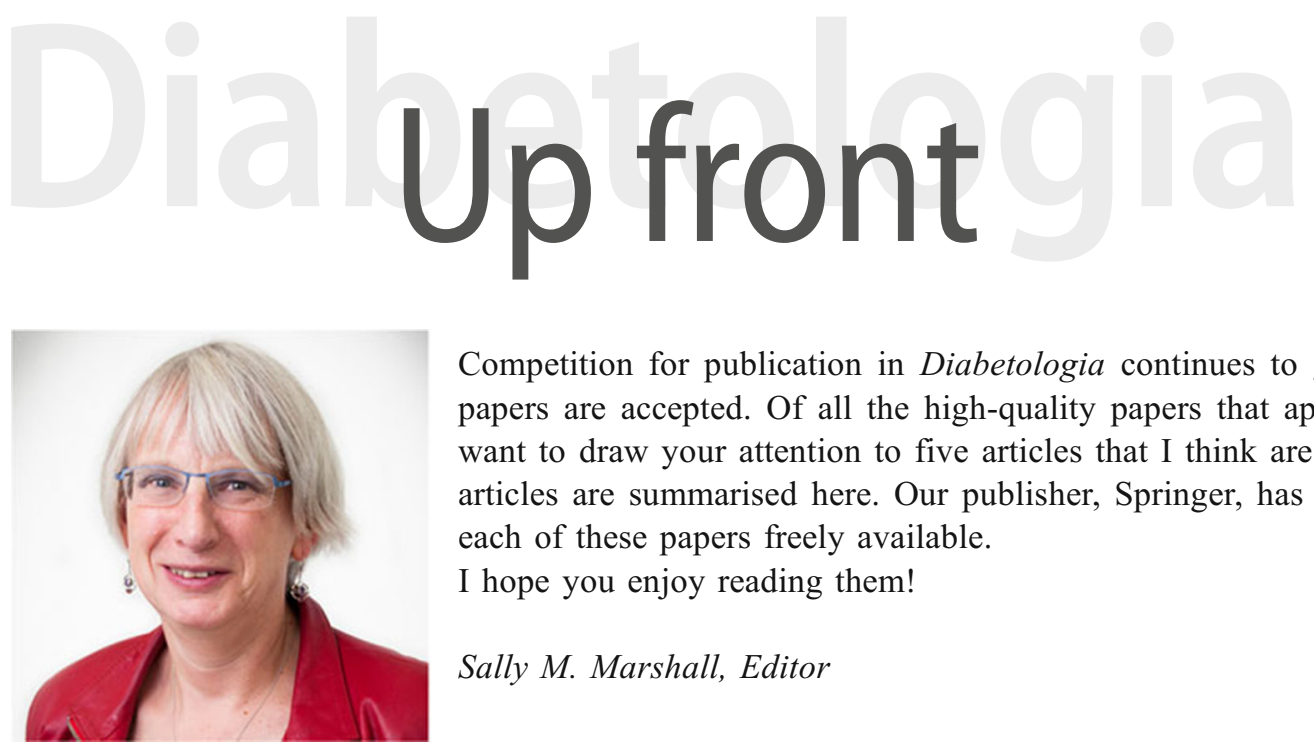

Competition for publication in Diabetologia continues to grow, and less than $20 \%$ of papers are accepted. Of all the high-quality papers that appear in this month's issue I want to draw your attention to five articles that I think are particularly interesting. The articles are summarised here. Our publisher, Springer, has kindly made the full text of each of these papers freely available.

I hope you enjoy reading them!

Sally M. Marshall, Editor

\section{Global trends in diabetes complications: a review of current evidence}

$\overline{\text { Jessica L. Harding, Meda E. Pavkov, Dianna J. Magliano, }}$ Jonathan E. Shaw, Edward W. Gregg

As the prevalence of diabetes continues to increase around the world, the profile of diabetes complications is likely to change. In this issue, Harding et al (https://doi.org/10.1007/ s00125-018-4711-2) summarise the available evidence on current trends in diabetes complications. Overall, data primarily from high-income countries report that rates of amputations, acute complications, cardiovascular disease and mortality among people with diabetes are declining. In spite of this, people with diabetes remain at significantly higher risk for these complications compared with those without diabetes. Notable declines in mortality, coinciding with increased survival, may lead to proportional increases in other forms of morbidity (e.g. renal disease, infections, cancers and physical and cognitive disability), with important implications for the clinical and public health burden of diabetes. The continued monitoring of global trends in diabetes complications, particularly in low- and middle-income countries, will be essential to understanding the burden of diabetes moving into the future.

(d) The figures from this review are available as a downloadable slideset.
Dynamics of adipose tissue turnover in human metabolic health and disease

Ursula White, Eric Ravussin

The magnitude of adipose tissue mass is determined by dynamic changes in the synthesis and breakdown (i.e. turnover) of adipocytes and triacylglycerols. Obesity is characterised by excessive adiposity and is a risk factor for many cardiometabolic disorders, including the metabolic syndrome and type 2 diabetes. Evidence suggests that the manner of subcutaneous adipose expansion (hypertrophy vs hyperplasia), as well as adipocyte death, can contribute to the pathogenesis of obesity-related disorders. Despite the plausible role of adipose turnover in human health and pathology, little is known about the in vivo kinetics of adipose tissue components. In this issue, White and Ravussin (https://doi.org/10.1007/s00125-0184732-x) provide an overview of current in vivo approaches that are being implemented to assess the turnover of adipose cells and triacylglycerols, namely, ${ }^{2} \mathrm{H}$ labelling and ${ }^{14} \mathrm{C}$ dating. In addition, the authors summarise findings from in vitro studies and discuss the role of adipose tissue turnover in metabolic health and disease in humans.

(b) The figures from this review are available as a downloadable slideset. 
Disruption of beta cell acetyl-CoA carboxylase-1 in mice impairs insulin secretion and beta cell mass

James Cantley, Aimee Davenport, Laurène Vetterli, Nandor J. Nemes, P. Tess Whitworth, Ebru Boslem, Le May Thai, Natalie Mellett, Peter J. Meikle, Kyle L. Hoehn, David E. James, Trevor J. Biden

The role of glucose oxidation in triggering insulin secretion from pancreatic beta cells is well characterised. Glucose also drives anaplerotic flux, leading to the production of malonylCoA by the enzyme acetyl-CoA carboxylase 1 (ACC1), yet the role of $\mathrm{ACC} 1$ in beta cell function in vivo is not clear. In this issue, using tissue-specific ACC1 gene (Acaca) knockout mouse models and a combination of in vivo and ex vivo experiments, Cantley et al (https://doi.org/10.1007/s00125-0184743-7) report that beta cell ACC1 activity is necessary for normal insulin secretion profiles and whole body glucose homeostasis. Moreover, they reveal that long-term loss of beta cell ACC1 leads to reductions in beta cell size and levels of enzymes involved in protein synthesis. These results demonstrate the importance of the ACC1-coupled pathway in sustaining adequate beta cell growth and function and suggest that potential strategies to enhance this pathway could promote a functional beta cell mass during diabetes.

\section{A cost analysis of intensified vs conventional multifactorial therapy in individuals with type 2 diabetes: a post hoc analysis of the Steno-2 study}

Joachim Gade, Jens Oellgaard, Rikke Ibsen, Peter Goede, Emil Nørtoft, Hans-Henrik Parving, Jakob Kjellberg, Oluf Pedersen

Intensified multifactorial therapy in type 2 diabetes patients with microalbuminuria increased median life span by 7.9 years and delayed incident cardiovascular disease by 8.1 years in the Steno-2 Study. However, the economic implications of this approach are unclear. In this issue, Steno-2 study investigators (https://doi.org/10.1007/s00125-018-4739-3) report that, over 21.2 years of follow-up, there was no significant difference in total direct medical costs between the intensive-therapy group vs the conventional therapy group. Furthermore, they showed a reduced cost per person-year in the intensive-therapy group. Cost of medications was highest with intensive therapy, but this additional cost was offset by the reduction in expenses for inpatient admissions for late complications and primary healthcare costs with conventional therapy. These findings emphasise that investment in early intensified multifactorial therapy may lead to an initial increase in healthcare expenditure; this investment appears, however, to be returned over time by health benefits and increased longevity.

Basal fatty acid oxidation increases after recurrent low glucose in human primary astrocytes

Paul G. Weightman Potter, Julia M. Vlachaki Walker, Josephine L. Robb, John K. Chilton, Ritchie Williamson, Andrew D. Randall, Kate L. J. Ellacott, Craig Beall

Hypoglycaemia is a major limiting factor for good glycaemic control in diabetes. Brain glucose-sensing mechanisms are incompletely understood and the contribution of glia, such as astrocytes, to hypoglycaemia detection and defective glucose counter-regulation requires more study. In this issue, Weightman Potter et al (https://doi.org/10.1007/s00125-0184744-6) characterise the intrinsic mechanisms by which human primary astrocyte function is altered by acute and recurrent low glucose (RLG) exposure in vitro. They report that astrocytic AMP-activated protein kinase (AMPK) is activated by physiologically relevant reductions in glucose concentration. In addition, they found that basal fatty acid oxidation rates were increased by RLG, as were markers of mitochondrial dysfunction. Glycolytic rates were enhanced after RLG exposure, but this was not due to increased glucose uptake, nor did this lead to increased glycogen content. Moreover, following a recovery period, aspects of mitochondrial function were restored. These novel observations demonstrate that human primary astrocytes adapt to RLG to successfully maintain intracellular nucleotide levels. These data suggest the potential involvement of glial mitochondrial adaptations in defective glucose counterregulation or protection against hypoglycaemia. Studies are needed to further elucidate interactions between neurons and glial cells during and after recurrent hypoglycaemia.

All text supplied by the authors. 